\title{
The Markov Model of Bean Optimization Algorithm and Its Convergence Analysis
}

\author{
Xiaoming Zhang, Halei Wang, Bingyu Sun, Wenbo Li, Rujing Wang \\ Institute of Intelligent Machines, Chinese Academy of Sciences, Hefei, Anhui Province, 230031, China
}

\author{
Received 20 October 2011 \\ Accepted 5 December 2012
}

\begin{abstract}
By simulating the self-adaptive phenomena of plants in nature, a novel evolutionary algorithm named Bean Optimization Algorithm (BOA) was proposed in 2008. BOA can be used for resolving complex optimization problems. As BOA is a new optimization algorithm, theoretical analysis of the algorithm is still very preliminary. Research on the state transfer process and the convergence behavior of BOA is of great significance for understanding it. In this paper, we build the Markov chain model of this algorithm and analyze the characters of this Markov chain. Then we analyze the transferring process of the bean memeplex status series and point out that the memeplex status series will enter the best status set. We also prove that this algorithm meets the requirement of global convergence criterion of random search algorithms. Finally we get the conclusion that BOA will make sure to get the global optimum.
\end{abstract}

Keywords: swarm intelligence; bean optimization algorithm; Markov chain; global convergence

\section{INTRODUCTION}

Many complex self-adaptive phenomena in nature often give us inspiration. For example, organisms and natural ecosystems can solve many highly complex optimization problems through their own evolutions. Some scholars have been inspired from these natural phenomena and many nature-inspired optimization algorithms have been proposed to solve complex optimization problems. The idea of the novel optimization algorithms which simulates the natural ecosystem mechanisms is different from the idea of the classic optimization algorithms. Their appearances greatly enrich the optimization technology and bring new life and hope for the solution of complex optimization problems which are difficult to deal with by traditional optimization methods.

Nature-inspired optimization algorithm refers to the computing technology and algorithms which based on the functions, characteristics and mechanism of the nature to solve the optimization problems, such as genetic algorithms
(GA) [1], particle swarm optimization (PSO) [2], artificial fish-swarm algorithm [3], free search algorithm [4], human evolution model algorithm [5], group search optimization algorithm [6], shuffled frog leaping algorithm [17], etc. Because the structure of nature biology is complex and sophisticated, they have a high degree of adaptive capacity and strong collaborative capabilities both in the evolutions and behaviors. Through collaboration, they can get the best environment for survival. Therefore, most of the natureinspired algorithms have the character of self-organizing, self-adaptive and self-learning. When solving some complex problems which could not be solved easily by the traditional optimization algorithm, the nature-inspired optimization algorithms have its own unique advantages.

At present, the nature-inspired optimization algorithms have been used to solve complex optimization problems in many fields successfully, for example in task assignment [7], classification [8], TSP [18], cluster analysis [19], scheduling [20] and gene selection [9].

However, because the theoretical basis of nature-inspired optimization algorithms comes from the model of biological 
communities in nature, the relevant mathematical analysis is still quite weak. This leads to many problems in the current study, such as: universal theoretical analysis of intelligent algorithms, theoretical basis of parameter settings of intelligent algorithms.

In 2008, inspired by the transmission mode of seeds, a novel evolutionary algorithm named Bean Optimization Algorithm (BOA) was proposed [10], which could be used to solve complex optimization problems by simulating the adaptive phenomenon of plants in nature. BOA is the combination of nature evolutionary tactic and limited random search. It has a stable robust behavior on explored tests and stands out as a promising alternative to existing optimization methods for engineering designs or applications. At present, two algorithm models have been constructed for BOA, including piecewise function model and normal distribution model. BOA has been successfully applied in solving of TSP with ACO [11], materials scheduling and earthquake recovery [12] and reconstruction planning of China [13].

As BOA is a new nature-inspired optimization algorithm, theoretical analysis of the algorithm is still very preliminary. Research on the state transfer process and the convergence behavior of BOA is of great significance for understanding it. In this paper, the Markov chain model of BOA is established to analyze the behavior of the population state transfer process. Based on the model, the convergence analysis is carried out.

\section{Bean Optimization Algorithm (BOA)}

\subsection{Basic idea of $\mathrm{BOA}$}

As we know, there are many different modes of transmission of seeds in nature. For example, most of the legume explore scattering mode. The scattering mode can be described as the following. When the beans are ripe, their skin becomes dry and hard in the sun's radiation. Then their skin bursts. Most beans are ejected to the region around the plant. Also, some beans may move or fly far away from the plant for some reasons, for example, carried by wind or animals. Assume that all beans will then grow and develop in the region where they land on. There is no doubt that some beans will grow to be very strong and produce more beans where the region they land on is very fertile. On the contrary, some may soon be phased out because they are not suitable to grow in the region where they grow on. After a long time, a large number of beans will be gathered in the fertile region, and most of those beans in the infertile regions will be disappearing. Inspired by this phenomenon, in this paper, the domain of optimization problem will be treated as a land and the position of the target point is set in the most fertile position. The degree of the region's fertility is determined in accordance with the objective function values. It can be given a concrete example: when a packet of beans are randomly spread to the land, the probability of their growing vigorously and producing more offspring will be high if the beans fall in fertile region. If the beans fall in the barren region, then they may become extinct in the future. After the beans have evolved for several generations, there might be one or several plants growing in the most fertile region. In $\mathrm{BOA}$, the evolution process is abstracted. It can be described as such phenomenon: in every new generation, most of the descendant plants emerged around several most robust parent plants (we call the robust parent plants father beans).

\subsection{Algorithm Design}

In BOA, individual beans are expressed with real number vector like

$$
X=\left\{x_{1}, x_{2}, x_{3}, \ldots, x_{n}\right\},
$$

where $n$ is the dimension which is determined by the scale of problem to be resolved. The bean group is composed of a large number of beans. And the size of the bean group can be adjusted according to the practical situations. In addition, beans are sown to the region which is defined by the problem. Father beans are those beans whose fitness values are larger than most of others. In BOA, the number of descendant beans and the parameters of distribution will be set according to their father bean's fitness value. That is to say the larger is the value of father bean's fitness, the larger is the number of its descendants and the closer is the distance between them. Otherwise, the number of its descendants will be fewer and its descendants will have a more random distribution. The basic equation of beans is shown as follows:

$X[i]=\left\{\begin{array}{l}X[i], \text { if } X[i] \text { is a father bean } \\ \text { Distribution }\left(X_{m b}\right) \times A, \text { if } X[i] \text { is not a father bean }\end{array}\right.$

$X[i]$ is the position of bean $i$ and $X_{m b}$ is the position of father bean of bean $i$. Parameter $A$ can be set according to the range of problem to be resolved. Distribution $\left(X_{m b}\right)$ is the distribution function of father bean in order to get the positions of its descendants.

Normal distribution is an important family of continuous probability distributions, which has been applied in many fields [14]. The importance of the normal distribution as a model of quantitative phenomena in the natural is due in part to the central limit theorem. It is far more common to 
describe a normal distribution by its mean $\mu$ and variance $\sigma^{2}$. The probability density function in a convenient standard form is shown as:

$$
f(X)=\frac{1}{\sigma \sqrt{2 \pi}} e^{-(X-\mu)^{2} /\left(2 \sigma^{2}\right)},-\infty<X<+\infty .
$$

Many measurements, ranging from psychological to physical phenomena can be approximated, to varying degrees, by the normal distribution. While the mechanisms underlying these phenomena are often unknown, the use of the normal model can be theoretically justified by assuming that many small, independent effects are additively contributing to each observation. So in this paper, we also adopt normal distributions as the distribution functions of beans. The mean and variance of every distribution functions are set according to the fitness of father beans.

In addition, the distribution of some beans does not follow the equation discussed above. According to the distribution of their father beans, they choose a random position in order to reinforce the global optimization performance.

When the descendant beans finish locating, their fitness values will be evaluated. The beans with the most optimal fitness value will be selected as the candidates of next generation father beans. The father beans candidates should also satisfy the condition that the distance between every two father beans must be larger than the distance threshold. This condition assures that the father beans can have a fine distribution to avoid premature convergence and to enhance the performance of global optimization. If all the conditions can be satisfied, then this descendant bean can be set as the next generation father bean. The flow chart of father beans selection is shown in Fig.1.

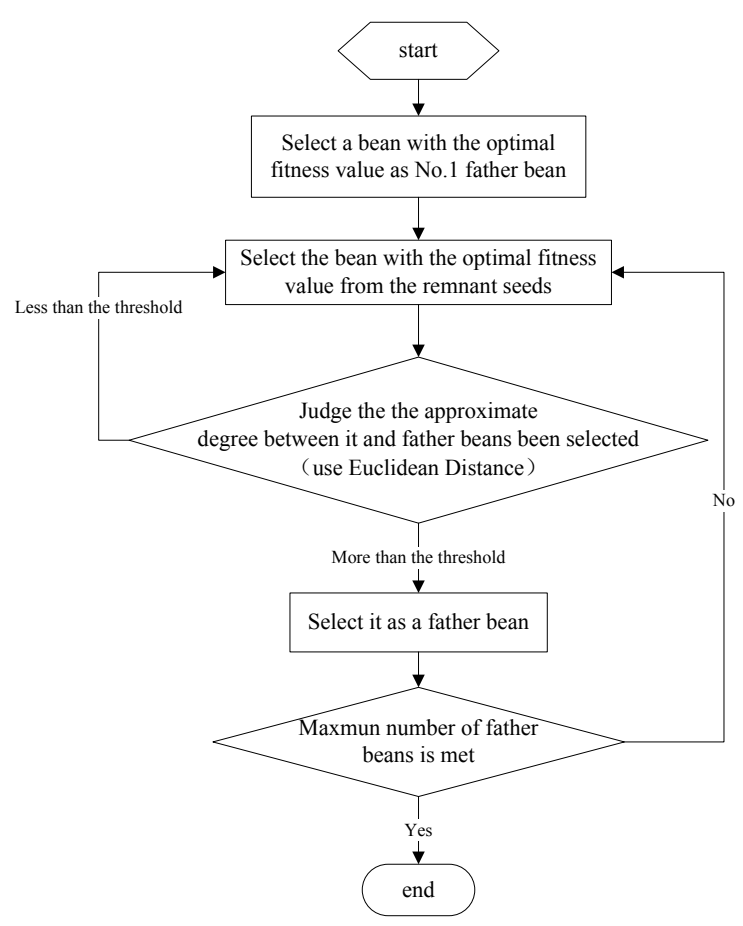

Fig. 1 the Selection of Father Beans

According to the beans evolution equations, beans are sown in the radiate area of their father beans in each generation. Evolution will go on until the desired optimization result is obtained or the No.1 father bean (the optimal bean in all generations) does not change anymore. The flow chart of the Algorithm is shown in Fig.2.

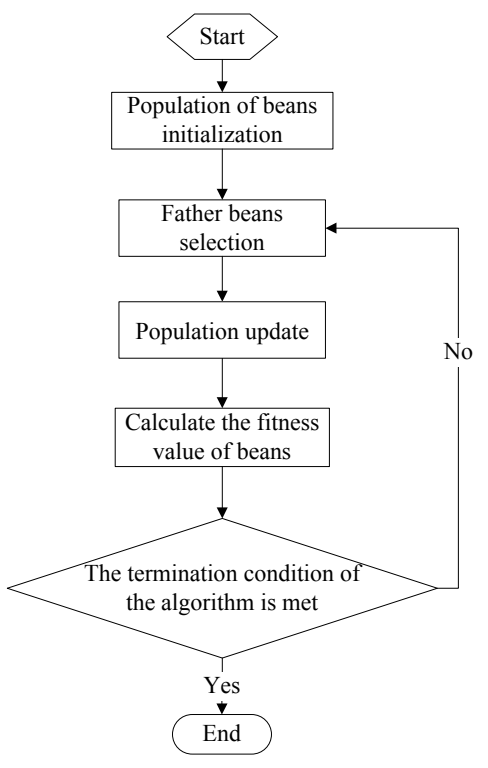

Fig.2 the Flow Chart of BOA 


\section{BOA's Markov Model and Convergence Analysis}

\subsection{Convergence criterion}

BOA is a kind of stochastic optimization algorithms. Thus, we can use the theory about how stochastic algorithm can converge to the global optimization solution with probability one. We use the theory which has been proved to be right by Solis and Wets [15]. Here, we list their main conclusions without proof.

We make the following settings: $\boldsymbol{A}$ is the feasible solution space. $f$ is The fitness function. $\langle A, f\rangle$ is the optimization problem. $\mathrm{D}$ is the optimization algorithm. $\zeta$ is the solution which has been searched by the algorithm $D$. If the result of $k$-th iteration is $x_{k}$, the result of the next iteration is $x_{k+1}=D\left(x_{k}, \zeta\right)$.

The infimum of feasible solution space in the Lebesgue measure space is set as followings:

$$
\alpha=\inf \{t \mid v(x \in \boldsymbol{A} \mid f(x)<t)>0\}
$$

$v(\boldsymbol{X})$ is the Lebesgue measure on set $\boldsymbol{B} . R_{\varepsilon, M}$ is the region contains the optimal solutions :

$$
R_{\varepsilon, M}=\left\{\begin{array}{rc}
\{x \in A \mid f(x)<\alpha+\varepsilon\}, & \alpha \text { is finite } \\
\{x \in A \mid f(x)<-C\}, & \alpha=-\infty
\end{array}\right.
$$

Thereinto, $\varepsilon>0, \quad C$ is a large positive number.

(Hypothesis 1) D s.t. $\left\{f\left(\mathrm{x}^{\mathrm{k}}\right)\right\} \underset{k=0}{\infty}$ nonincreasing

$$
\begin{array}{r}
f(D(x, \zeta)) \leq f(x), \zeta \in \boldsymbol{A} \\
\Rightarrow f(D(x, \zeta)) \leq \min \{f(x), f(\zeta)\}
\end{array}
$$

(Hypothesis 2) Zero probability of repeatedly missing any positive-volume subset of $\mathrm{S}$.

$$
\forall \boldsymbol{B} \in \boldsymbol{A}, \quad \text { s.t.v }(\boldsymbol{B})>0, \sum_{k=0}^{\infty}\left(1-u_{k}(\boldsymbol{B})\right)=0
$$

$\boldsymbol{B}$ is the $\sigma$-algebra of subset of $\mathrm{R}^{\mathrm{n}} \cdot u_{k}(\boldsymbol{B})$ is a probability measure of the result of k-th iteration on set $\boldsymbol{B}$.

Theorem 1 (Global Search Convergence Theorem). Suppose $f$ measurable, $A \subseteq R^{n}$ measurable, (Hypothesis 1), (Hypothesis 2), and $\left\{x^{k}\right\}_{k=0}^{\infty}$ generated by the algorithm. Then

$$
\lim _{k \rightarrow \infty} P\left(x_{k} \in R_{\varepsilon, M}\right)=1
$$

$P\left(x_{k} \in R_{\varepsilon, M}\right)$ is the probability that $x_{k}$ belongs to $\mathrm{R}_{\varepsilon}$.

\subsection{The Mathematical definition of basic concepts of BOA}

Definition 1 (Beans' individual states and individual state space) The individual states of beans are composed of the locations of individual bean and its father beans (they are denoted by $p s$ ). It is denoted by $u=(x, p s), x \in \boldsymbol{A}, p s \in \boldsymbol{A}$ and $\boldsymbol{A}$ is the feasible solution space. The set which is composed of all possible individual states of the beans is called individual state space. It is denoted by $U=\{u=(x, p s) \mid x \in A, p s \in A, f(p s) \leq f(x)\}$.

Definition 2 (Population state and population state space) Population state, which is denoted by $s=\left(u_{1}, u_{2}, \ldots, u_{n}, p s, p b\right)$, is composed of all the individual states of beans, the states of the parent beans and the best individual state of the population (they are denoted by $p b$ ). The beans are sorted according to their fitness value in every iterations of BOA. Therefore, $f(p b) \leq f(p s) \leq f\left(x_{i}\right), 1 \leq i \leq n$.

The population state space of BOA is the set of all possible populations. It is denoted by $S=\left\{s=\left(u_{1}, u_{2}, \ldots, u_{n}, p s, p b\right) \mid u_{i} \in U, 1 \leq i \leq n\right\}$ and $n$ is the number of individual beans in a population.

\subsection{The Markov model of $\mathrm{BOA}$}

Definition 3 (individual state transition of BOA) For $\forall u_{i} \in U$ and $\forall u_{j} \in U$ in BOA, individual state transition is denoted by $T_{u}\left(u_{i}\right)=u_{j}$ which means the individual state $u_{i}$ transferred to $u_{j}$ by one step.

Theorem 2 In BOA, the probability of the individual state $u_{1}$ transferred to $u_{2}$ by one step which is denoted by $P\left(T_{u}\left(u_{1}\right)=u_{2}\right)$ can be expressed as formula (5).

Proof: Based on definition 3, we can get that:

$$
p\left(T_{u}\left(u_{1}\right)=u_{2}\right)=p\left(x_{1} \rightarrow x_{2}\right) * p\left(p s_{1} \rightarrow p s_{2}\right)
$$

Based on formula (1) and formula (2) of BOA, we can get the formula about $p\left(x_{1} \rightarrow x_{2}\right)$ and $p\left(p s_{1} \rightarrow p s_{2}\right)$.

$$
\begin{gathered}
P\left(x_{1} \rightarrow x_{2}\right)= \begin{cases}\frac{1}{\sigma \sqrt{2 \pi}} e^{-\left(x_{2}-p s_{1}\right)^{2} /\left(2 \sigma^{2}\right)} & f\left(x_{1}\right)<f\left(p s_{1}\right) \& \& P_{\text {trans }}<P_{\text {threshold }} \\
\frac{1}{|\boldsymbol{X}|} & f\left(x_{1}\right)<f\left(p s_{1}\right) \& \& P_{\text {trans }} \geq P_{\text {threshold }}\end{cases} \\
P\left(p s_{1} \rightarrow p s_{2}\right)= \begin{cases}1 & f\left(p s_{2}\right) \leq f\left(p s_{1}\right) \\
0 & f\left(p s_{2}\right)>f\left(p s_{1}\right)\end{cases}
\end{gathered}
$$


In the above formula, $\mathrm{ps}_{1}$ and $\mathrm{ps}_{2}$ are the states of their father beans. $P_{\text {trans }}$ is a random number between 0 and 1 . $P_{\text {threshold }}$ is the threshold of population variation. Because $\mathrm{ps}_{i}$ and $x_{i}$ are multi-dimensional data, the sign of plus and minus indicates the vector addition and subtraction. Absolute value denotes the volume of hyperspace cubic. That is to say, $|X|$ denotes the size of the state space of beans.

Definition 4 (Population state transition) For $\forall s_{i}=\left(u_{i 1}, u_{i 2}, \ldots, p s_{i}, p b\right) \in S, \quad \forall s_{j}=\left(u_{j 1}, u_{j 2}, \ldots, \mathrm{ps}_{j}, \mathrm{pb}\right) \in S$ in BOA, population state transition is denoted by $T_{s}\left(s_{i}\right)=s_{j}$ which means the population state $s_{i}$ transferred to $s_{j}$ by one step.

Theorem 3 In BOA, the probability of the population state $s_{i}$ transferred to $s_{j}$ by one step which is denoted by $P\left(T_{s}\left(s_{i}\right)=s_{j}\right)$ can be expressed as formula (8).

$$
P\left(T_{s}\left(s_{i}\right)=s_{j}\right)=\prod_{k=1}^{N} P\left(T_{u}\left(u_{i k}\right)=u_{j k}\right)
$$

Proof: Because population $s_{i}=\left(u_{i 1}, u_{i 2}, \ldots, p s_{i}, p b\right)$ should transferred to population $s_{j}=\left(u_{j 1}, u_{j 2}, \ldots, p s_{j}, p b\right)$ by one step, the states of all the beans, include all the parent beans, should transferred to the corresponding states in $s_{j}$. According to the Population states update mechanism of BOA, the positions of all the beans will change at each iterations of the algorithm. So we can get that:

$$
T_{u}\left(u_{i 1}\right)=u_{j 1} T_{u}\left(u_{i 2}\right)=u_{j 2,}, \ldots, T_{u}\left(u_{i n}\right)=u_{j n}
$$

$$
\begin{aligned}
P\left(T_{s}\left(s_{i}\right)=s_{j}\right) & =P\left(T_{u}\left(u_{i 1}\right)=u\right) P\left(T_{u}\left(u_{i 2}\right)=u_{j 2}\right) \ldots P\left(T_{u}\left(u_{i N}\right)=u_{j N}\right) \\
& =\prod_{k=1}^{N} P\left(T_{u}\left(u_{i k}\right)=u_{j k}\right)
\end{aligned}
$$

Concluded.

Theorem 4 In BOA, the sequence of population state $\{S(t): t \geq 0\}$ is finite homogeneous Markov chain.

Proof: In the bean state space $\mathrm{u}$, the locations $x$ of the beans are finite. So the bean state space $u$ is finite. Every population $\mathrm{s}$ is composed of $\mathrm{n}$ beans. The number of $\mathrm{ps}_{i}$ and $\mathrm{pb}$ is obviously finite. Therefore, the population state space $S$ is finite, that is to say, the sequence of population state $\{S(t): t \geq 0\}$ is finite. According to Theorem 3, $\forall S(t-1) \in \boldsymbol{S}, \forall S(t) \in \boldsymbol{S}$, its transfer probability $P\left(T_{s}(S(t-1))=S(t)\right)$ is determined by the probability of individual state transition $\prod_{i=1}^{n} P\left(T_{u}\left(u_{i, t-1}\right)=u_{i, t}\right)$. Based on

Theorem 2, the probability of individual state transition is determined by the locations of individual bean and its father beans: $P\left(x_{t-1} \rightarrow x_{t}\right)$ and $P\left(p s_{t-1} \rightarrow p s_{t}\right) \cdot P\left(x_{t-1} \rightarrow x_{t}\right)$ and $P\left(p s_{t-1} \rightarrow p s_{t}\right)$ are determined by $x_{t-1}, p s_{t-1}$. So, $P\left(T_{s}\left(S_{t-1}\right)=S_{t}\right)$ is only determined by the individual states of beans at time $t-1$. So the sequence of population state $\{S(t): t \geq 0\}$ is finite Markov chain.

We can see from the formula (6) and formula (7) that $P\left(x_{t-1} \rightarrow x_{t}\right)$ and $P\left(p s_{t-1} \rightarrow p s_{t}\right)$ are nothing to do with time $t$-1. That is to say, $P\left(T_{u}\left(u_{t-1}\right)=u_{t}\right)$ has no relationship with time $t$ - 1 . So $\{S(t): t>0\}$ is homogeneous.

So the sequence of population state $\{S(t): t \geq 0\}$ is finite homogeneous Markov chain.

\subsection{Convergences Analysis of $\mathrm{BOA}$}

For the convenience of analysis, we first analyze the BOA contains only one population. So in the following analysis, $p b=p s$.

Definition 5 (the set of the optimal state of the individual) Let's Suppose that $p b$ is the global optimal solution of the optimization problem $\langle\boldsymbol{A}, f\rangle$. The Set of the optimal state of the individual is $B=\{u=(x, p s) \mid f(p s)=f(p b), u \in U\}$. $B \subseteq U$.

Theorem $5 B$ is a closed set on the individual state space $U$. Proof: $\forall U_{i} \in B, \forall U_{j} \notin B$. According to Theorem 2,

$$
p\left(T_{u}\left(u_{i}\right)=u_{j}\right)=p\left(x_{i} \rightarrow x_{j}\right) * p\left(p_{i} \rightarrow p_{j}\right) .
$$

Because $U_{i} \in B, U_{j} \notin B$, according to Definition $5, f\left(p s_{j}\right) \geq f\left(p s_{i}\right)=f(p b)=\inf (f(a)), a \in A$. According to formula (7),

$P\left(\mathrm{ps}_{t-1} \rightarrow \mathrm{ps}_{t}\right)=\left\{\begin{array}{ll}1 & f\left(\mathrm{ps}_{t}\right) \leq f\left(\mathrm{ps}_{t-1}\right) \\ 0 & f\left(\mathrm{ps}_{t}\right)>f\left(\mathrm{ps}_{t-1}\right)\end{array}\right.$.

We can get that $p\left(p_{i} \rightarrow p_{j}\right)=0$. That is to say that $p\left(T_{u}\left(u_{i}\right)=u_{j}\right)=0$. So $\mathrm{B}$ is a closed set on the individual state space $U$.

Definition 6 (Set of the optimal state of the population) Let's Suppose that $p b$ is the global optimal solution of the optimization problem $\langle\boldsymbol{A}, f\rangle$. The Set of the optimal state of the population is $F=\left\{\left(u_{1}, u_{2}, \ldots u_{n}\right) \mid \exists u_{i} \in B, 1 \leq i \leq n\right\}$.

Theorem 6 For the sequence of population state $\{S(t): t \geq 0\}, \mathrm{F}$ is a closed set on the population state space $S$. 
Proof: $\forall s_{i} \not \subset F, \forall s_{j} \subset F$. According to Theorem 3,

$$
P\left(T_{s}\left(S_{j}\right)=S_{i}\right)=\prod_{n=1}^{N} P\left(T_{u}\left(u_{j n}\right)=u_{i n}\right) .
$$

If $T_{s}\left(S_{j}\right)=S_{i}$ is true, there must be:

$$
T_{u}\left(u_{j n}\right)=u_{i n}, u_{j n} \in B, u_{i n} \notin B, 1 \leq n \leq N .
$$

According to Theorem 5, $B$ is a closed set on the individual state space $U$. Then $p\left(T_{u}\left(u_{j n}\right)=u_{i n}\right)=0$. That is to say, $P\left(T_{s}\left(S_{j}\right)=S_{i}\right)=0$. So $F$ is a closed set on the population state space $S$.

According to Definition 5, $f\left(p s_{j}\right) \geq f\left(p s_{i}\right)=f(p b)=\inf (f(a)), a \in A$. According to formula (7),

$$
P\left(p s_{t-1} \rightarrow p s_{t}\right)=\left\{\begin{array}{ll}
1 & f\left(p s_{t}\right) \leq f\left(p s_{t-1}\right) \\
0 & f\left(p s_{t}\right)>f\left(p s_{t-1}\right)
\end{array} .\right.
$$

We can get that $p\left(p_{i} \rightarrow p_{j}\right)=0$. That is to say that

$$
p\left(T_{u}\left(u_{i}\right)=u_{j}\right)=0 \text {. }
$$

So $B$ is a closed set on the individual state space $U$.

Theorem 7 There is no closed set $G$ in the population state space, which makes $F \cap G=\phi$.

Proof: The apagoge will be used to prove this theorem. Let's suppose that there is a closed set $G$ in the population state space, which can make $F \cap G=\phi$.

Firstly, we hypothesize $\exists S_{i}=\left(u_{i}, u_{i}, \ldots u_{i}, u_{i}\right) \in F$, $\forall S_{j}=\left(u_{j 1}, u_{j 2}, \ldots, p s_{j}, p s_{j}\right) \in G \quad, \quad f\left(u_{i}\right)=p b \quad$, $f\left(p s_{j}\right)>f\left(u_{i}\right) . l$ is the transfer step. $\forall l, l \geq 1$, according to Chapman-Kolmogorov equation, the probability of the population state $s_{j}$ transferred to $s_{i}$ by $l$ steps is :

$$
P_{S_{j}, S_{i}}^{l}=\sum_{S_{\eta} \in S} \ldots \sum_{S_{\eta_{-1}} \in S} P\left(T_{s}\left(S_{j}\right)=S_{r_{1}}\right) P\left(T_{s}\left(S_{r_{1}}\right)=S_{r_{2}}\right) \ldots P\left(T_{s}\left(S_{r_{l_{1}}}\right)=S_{i}\right)
$$

If $l=1$, there must be $P\left(T_{s}\left(S_{j}\right)=S_{i}\right)$ in the expression of $P_{S_{j}, S_{i}}^{l}$. According to the Theorem 3,

$$
P\left(T_{s}\left(S_{j}\right)=S_{i}\right)=\prod_{n=1}^{N} P\left(T_{u}\left(u_{j n}\right)=u_{i n}\right) \cdot
$$

Because $s_{i} \in F$ and $s_{j} \notin F$, that means every individuals transferred from current status to the optimal status. So $\prod_{n=1}^{N} P\left(T_{u}\left(u_{j n}\right)=u_{i n}\right)=1 \neq 0$, that is to say, $P_{S_{i}, S_{j}}^{l} \neq 0$. Therefore a contradiction occurs between the conclusion and the premise. So the set $G$ is not a closed set. Theorem 7 is proved.

Theorem 8 [16] Assume that there is a closed set $C$ in the Markov chain and there is no other closed set D which satisfies that $C \cap D=\phi$. So,

$$
\begin{cases}\lim _{n \rightarrow \infty} P\left(X_{n}=j\right)=\pi_{j} & j \in C \\ \lim _{n \rightarrow \infty} P\left(X_{n}=j\right)=0 & j \notin C\end{cases}
$$

Theorem 9 In BOA, when the number of iteration goes to infinity, the sequence of population state $\{S(t): t \geq 0\}$ must enter the set of the optimal state of the population.

Proof: according to Theorem 6, Theorem 7 and Theorem 8, this conclusion can be got.

Theorem 10 BOA will converge to the global optimal solution with probability 1 .

Proof: In BOA, the best position in the population is retained at every iterations because the No.1 father bean is always the bean with the best fitness value. So algorithm satisfies the convergence condition (Hypothesis 1) in the convergence criterion.

According to theorem 9, the probability that BOA can not find the best solution in approximate infinite search iterations is 0 . That is to say, BOA satisfies the convergence condition (Hypothesis 2) in the convergence criterion.

Therefore, according to Global Search Convergence Theorem (Theorem 1), BOA will converge to the global optimal solution with probability 1 .

In the above, we just analysis the BOA with one group. If groups are one more, at least the group which contains the No.1 parent bean can be analyzed like above. So the BOA with one more group will also converge to the global optimal solution with probability 1 .

\section{Conclusions}

BOA is conceptually simple and convenient to handle a variety of optimization problems, which makes it particularly attractive for real world applications.

In this paper, the Markov chain model of BOA is established and the analysis of the Markov chain is made. Then we prove that BOA satisfies the two convergence conditions of the random search algorithms and it is a global convergence algorithm.

One of the most significant merits of BOA is that it provides an open framework to utilize research results of transmission mode of beans (seeds) to tackle hard optimization problems. After millions even billions of years of natural selection, adaptive behavior of plant distribution, especially in complex natural environments, has been verified as excellent by evolution. Research on the transmission mode of beans (seeds) provides many searching strategies to be incorporated into BOA for solving difficult optimization problems. This article is a macro-level analysis of BOA about the state transition conditions and convergence 
behavior, more micro-level analysis of BOA about population distribution by evolution need to be studied further in the future.

\section{ACKNOWLEDGMENT}

This work was supported by the National Science Foundation of China under Grant 61203373, 91024008, 41101516 and the Knowledge Innovation Program of the Chinese Academy of Sciences.

\section{REFERENCES}

[1] J. H. Holland. Adaption in Natural and Artificial Systems (The MIT Press, Cambridge, MA, 1975).

[2] Kennedy J, Eberhart R. C. Particle swarm optimisation, In Proceedings of IEEE International Conference on Neural Networks(Piscataway, NJ, 1995), pp.1942-1948.

[3] Li Xiaolei, Shao Zhijiang, Qian Jixin. An Optimizing Method Based on Autonomous Animats: Fish-swarm Algorithm. Systems Engineering-Theory \& Practice, 22(11), (2002), pp.32-38.

[4] Penev Kalin, Littlefair Guy. Free Search-a comparative analysis. Information Sciences, 172(1-2), (2005), pp.173-193.

[5] Oscar Montiela, Oscar Castillob, Patricia Melinb, Antonio Rodríguez Díazc, Roberto Sepúlvedaa. Human evolutionary model: A new approach to optimization. Information Sciences, 177(10), (2007), pp.2075-2098.

[6] He, S.,Wu, Q.H., Saunders, J.R. Group Search Optimizer: An Optimization Algorithm Inspired by Animal Searching Behavio. IEEE Transactions on Evolutionary Computation, Vol.13(5), (2009), pp. $973-990$

[7] Shinn-Ying Ho, Hung-Sui Lin, Weei-Hurng Liauh, Shinn-Jang Ho. OPSO: Orthogonal Particle Swarm Optimization and Its Application to Task Assignment Problems. IEEE Transactions on Systems, Man and Cybernetics, Part A, 38(2), (2008), pp.288-298.

[8] Souda T., Silva A., Neves A. Particle Swarm based Data Mining Algorithms for classification task. Parallel Computing, 30(5), (2004), pp.767-783.
[9] Shutao Li, Xixian Wu, Mingkui Tan. Gene selection using hybrid particle swarm optimization and genetic algorithm. Soft Computing, 12(11), (2008), pp.1039-1048.

[10] Xiaoming Zhang, Rujing Wang, Liangtu Song. A Novel Evolutionary Algorithm-Seed Optimization Algorithm. Pattern Recognition and Artificial Intelligence. 21(5), (2008), pp.677-681.

[11] Yunming Li. Solving TSP by an ACO-and-BOA-Based Hybrid Algorithm. In Proceedings of 2010 International Conference on Computer Application and System Modeling(Taiyuan, China, 2010), pp.189-192.

[12] Pengfei Wang, Youqing Cheng. Relief Supplies Scheduling Based on Bean Optimization Algorithm. Economic Research Guide,Vol.8, (2010), pp.252-253.

[13] Xiaoming Zhang, Bingyu Sun, Tao Mei, Rujing Wang, Post-disaster Restoration Based on Fuzzy Preference Relation and Bean Optimization Algorithm. In Proceedings of the $2^{\text {nd }}$ IEEE Youth Conference on Information, Computing and Telecommunications(Beijing, China, 2010), pp.271-274.

[14] Wei Zongshu. Probability Theory and Mathematical Statistics (Higher Education Press, Beijing, China, 2000).

[15] Solis F, Wets R. Minimization by random search techniques. Mathematics of Operations Research, 6(1), (1998), pp.19-30.

[16] Zhang Wenxiu, Leung Yee. Mathematical Foundation of Genetic Algorithm( Xi'an Jiaotong University Press, Xi'an, China, 2003).

[17] Chen Fang, Ling Wang. An effective shuffledfrog-leapingalgorithm for resource-constrained project scheduling problem. Computers \& Operations Research, 39(5), (2012), pp.890-901.

[18] Xiaoming Zhang, Kang Jiang, Hailei Wang, Wenbo Li, Bingyu Sun. An Improved Bean Optimization Algorithm for Solving TSP, Lecture Notes in Computer Science,Vol.7331, (2012), pp.261-267.

[19] Niknam, T., Amiri, B. An efficient hybrid approach based on PSO, $\mathrm{ACO}$ and k-means for cluster analysis. Applied Soft Computing, 10 (1), (2010), pp.183-197.

[20] L.Y.Tseng, S.C.Chen. A hybrid metaheuristic for the resourceconstrained project scheduling problem. European Journal of Operational Research, 175(2), (2006), pp.707-721 